\title{
Estimación del Costo de un Producto Incluyendo La Distribución al Cliente en Condiciones de Incertidumbre
}

\author{
Cost Estimation of a Product Including Distribution to the Customer in Uncertainty Conditions \\ Marcos Moya Navarro, PhD \\ Universidad Latina de Costa Rica, Costa Rica, marcos.moya @ulatina.cr
}

\begin{abstract}
Resumen-El costo unitario de un producto es una dimensión que hay que considerar, para que las empresas generen una ventaja competitiva. Existen diversos procedimientos estandarizados para calcular ese costo. Uno de tales procedimientos es el costo estándar. Sin embargo, este método determina el costo de un producto basado en ciertas condiciones de demanda, eficiencia, entorno económico y de otros factores, lo que produce que cada vez que alguna de esas condiciones cambie, el costo unitario de los productos también cambie.

El modelado de simulación es una herramienta que contribuye significativamente a la determinación del costo unitario de un producto cada vez que alguna de las condiciones enumeradas anteriormente presente variaciones a través del proceso de planificación de la producción.

El objetivo de este trabajo es presentar una metodología para determinar el patrón de probabilidad del costo unitario de un producto mediante simulación desde que el proceso de planificación de los requerimientos de capacidad (CRP) comienza hasta su distribución al cliente final. Esta distribución de probabilidad les servirá a las empresas para apoyar el proceso de toma de decisiones conociendo el valor más probable del costo unitario del producto hasta un valor pesimista del costo, con sus respectivas probabilidades asociadas.
\end{abstract}

Palabras Clave-Planificación de los Recursos de Manufactura, Cadena de Suministros, Simulación.

Abstract- The unit cost of a product is a dimension that must be considered for companies to generate a competitive advantage. There are several standardized procedures to calculate that cost. One of these procedures is the standard cost. However, this method determines the cost of a product based on certain conditions of demand, efficiency, economic environment, and other factors, which means that, whenever any of those conditions change, the unit cost of the product also changes.

Simulation modeling is a tool that contributes significantly to determining the unit cost of a product whenever any of the conditions listed above present variations through the production planning process.

The objective of this paper is to present a methodology to determine the probability distribution of the unit cost of a product through simulation from the beginning of the capacity requirement planning process (CRP) to its distribution to the final

Digital Object Identifier (DOI): http://dx.doi.org/10.18687/LACCEI2018.1.1.223 ISBN: 978-0-9993443-1-6

ISSN: $2414-6390$ client. This probability distribution will help companies to support the decision-making process by knowing not only the unit cost expected value, but also the pessimistic value of the cost, with their respective associated probabilities.

Keywords-Manufacturing Resources Planning, Supply Chain, Simulation

\section{INTRODUCCION}

De entre los distintos sistemas existentes en la actualidad, un sistema comúnmente empleado para determinar el costo de la producción es el Sistema de Planificación de los Recursos de Manufactura MRP II el cual realiza el cálculo de costos de la producción y desarrolla los estados financieros de una compañía. (Domínguez Machuca y otros) (1995) [7].

El sitio web Investopidia [3] indica que el sistema MRP II se usa ampliamente por sí mismo, pero también como un módulo de sistemas de planificación de recursos empresariales (ERP) más amplios. Establece que este es un sistema basado en computadora que puede crear cronogramas de producción detallados utilizando datos en tiempo real para coordinar la llegada de los materiales de los componentes con la disponibilidad de la máquina y mano de obra.

La Compañía MIME Enterprise [14] indica que el sistema MRP II es un sistema ERP especialmente diseñado para manufactura, el cual es un sistema automatizado de programación que continuamente calcula los tiempos en que las órdenes de producción comenzarán o se completarán basado en las cantidades de productos a producir, el proceso de producción y la capacidad de los recursos para llevar a cabo las operaciones. El autor también indica que al dividir el proceso en operaciones individuales y evaluar la capacidad de cada recurso, se calcula el orden y se actualiza el plan de carga para cada uno de los recursos disponibles.

El sitio web Bizfluent [15] establece que el sistema de planificación del requerimiento de materiales MRP se centra en la fabricación de los productos y enfoca los aspectos de programación y control de materiales de la producción a los

16 $^{\text {th }}$ LACCEI International Multi-Conference for Engineering, Education, and Technology: "Innovation in Education and Inclusion", 19-21 July 2018, Lima, Peru. 
pedidos realizados por los clientes. El autor indica que esto limita su capacidad para basar las previsiones de producción en la demanda y es lo que distingue al MRP del MRP II, que rastrea aspectos adicionales de la producción, como requisitos de personal, estimaciones financieras, pronósticos de demanda y planificación comercial. El autor establece que el MRP II es en realidad más integrador y está estratégicamente más orientado que MRP, considerando que va más allá del corto plazo para ponderar los impactos a mediano y largo plazo de todos los recursos de producción.

El sitio web smallbusiness.chron.com [9] indica que establecer precios apropiados para los productos es difícil, y no saber cuánto se está gastando hace el problema aún más difícil. El autor establece que saber cuánto cuesta fabricar cada unidad de un producto le ayuda a la empresa a ponerle un precio adecuado a sus productos para que pueda cubrir sus costos y obtener ganancias. Pero resalta que no es solo el costo de las materias primas lo que debe contar.

El autor también indica que cuando una empresa produce grandes cantidades de productos idénticos, puede calcular el costo unitario para hacer un seguimiento de sus gastos de fabricación. El monto total de sus costos fijos y variables constituye el costo total de fabricación. Estos costos se contabilizan a medida que sus productos avanzan en el proceso de fabricación. Al final de la ejecución de producción, puede usar los costos fijos y variables totales para calcular los costos de producción de su unidad. Al comparar los costos unitarios de tiradas de producción similares, puede determinar si los costos actuales superan los costos presupuestados.

El sitio web http://searchdatacenter.techtarget.com/es/ [16] establece que la Planificación de los Recursos de Manufactura MRP II es un método integrado de planificación operativa y financiera para las empresas de manufactura que funciona como extensión de la Planificación de los Requerimientos de Material de ciclo cerrado abreviado como CLMPR). Este sistema de planificación de los recursos de manufactura costea los productos basado en un sistema de costos estándar.

El sitio web https://www.accountingtools.com [1] define el cálculo de costos estándar como la práctica de sustituir un costo esperado por un costo real en los registros contables, y luego registrar periódicamente las variaciones que muestran la diferencia entre los costos esperados y los reales. El autor indica que el cálculo de costos estándar implica la creación de costos estimados (es decir, estándar) para algunas o todas las actividades dentro de una empresa. La razón principal para usar los costos estándar es que hay una serie de aplicaciones en las que es demasiado lento recopilar los costos reales, por lo que los costos estándar se utilizan como una aproximación cercana a los costos reales.
El sitio web http://www.monografias.com [8] define el costo estándar como la cantidad que, según la empresa, debería costar un producto o la operación de un proceso durante cierto período, sobre la base de ciertas condiciones de eficiencia, económicas y de otros factores.

Peirleitner, Andreas J; Altendorfer, Klaus; Felberbauer, Thomas (2017) [17] presentan un caso práctico de una empresa austriaca de ingeniería mecánica. La mejora del sistema de fabricación basada en simulación se aplica a una planta de fabricación de componentes. Los autores indican que, en el primer paso de mejora, las decisiones de inversión en capacidad estratégica se respaldan con el uso de la simulación. En el segundo paso, se realiza una optimización de los parámetros de planificación MRP para mejorar el nivel de servicio y el inventario. Además, se evalúa el efecto de las decisiones relacionadas con la capacidad relativas a la reducción del tiempo de configuración y al outsourcing dependiente de la carga. Los resultados de este estudio de simulación muestran que el nivel de servicio y el inventario se pueden mejorar significativamente mediante la optimización de los parámetros de planificación y la reducción de los tiempos de preparación. Además, el estudio muestra que la subcontratación dependiente de la carga es una alternativa viable a la inversión de capacidad.

Hung, Yi-Feng, Huang, Chuan-Che y Yeh, Ying (2013) [10] indican que en un entorno de fabricación contra inventario (MTS) que utiliza una planificación de las necesidades de materiales (MRP), la verificación de la capacidad de un programa maestro de producción (MPS) requiere una planificación de requisitos de capacidad (CRP) que se puede calcular fácilmente. Los autores establecen que la eficiencia de una red de cadena de suministro se ve afectada por su tiempo de entrega general, que incluye el tiempo dedicado al procesamiento de pedidos, la fabricación y el transporte.

Jinks, Stuart (2012) [12] indica cómo utilizar la simulación dinámica para estimar los costos unitarios y los recursos de fabricación puede ayudar en las decisiones de diseño. Se introduce una especificación de marco que integra el diseño asistido por computadora (CAD), la simulación de eventos discretos (DES) y la metodología de costo basado en actividades (ABC). El marco ayuda a un equipo de diseño a comprender las consecuencias de las decisiones de diseño en términos de costo unitario y recursos de fabricación, devolviendo los datos agregados del costo unitario y la fabricación, directamente al equipo de diseño, dentro del entorno de diseño. El marco puede predecir con precisión los recursos necesarios para cumplir una cadena de suministro para una tasa de producción específica, que pueden utilizar los ingenieros de manufactura para ayudar en la planificación de la producción. 
Waterman, R; Rubin, D; Thomas, N; Gelman, A (1999) [19] indican que las estimaciones de costos creíbles requieren la recopilación de información de alta calidad como entradas componentes. Los autores establecen que, en particular, uno de los beneficios del enfoque del modelo de simulación es que obliga al usuario a pensar mucho sobre sus suposiciones y a centrarse en qué exactamente es lo que necesita ser medido. Por lo tanto, puede proporcionar un medio para explorar conjeturas y sus consecuencias, de puntos de vista diferentes o incluso opuestos.

Baunton, M J (1985) [2] establece que el control de inventarios es un aspecto vital pero poco comprendido en muchas empresas del Reino Unido. Indica que, para gestionar el inventario de manera efectiva, se requiere una comprensión clara del entorno empresarial, junto con una evaluación precisa de todos los plazos de entrega relevantes. El autor resalta que se requiere la planificación a continuación en varios niveles consistentes con los niveles de control de gestión y la organización y que las sensibilidades y los cuellos de botella deben revisarse de forma realista.

Chen, Jhitang Steve (1981) [4] indica que con el fin de utilizar eficazmente un proceso de fabricación en un entorno de producción que se enfrenta con una mayor variedad y cantidad de productos, subconjuntos y componentes completos, muchas empresas utilizan sistemas de planificación y control de producción de bases de datos informáticas, que a menudo se llaman Sistemas de planificación de necesidades de materiales (MRP). El autor integra en la investigación las funciones de planificación de procesos y planificación de producción para permitir que los planes de proceso se vean influenciados por la capacidad de producción que estará disponible en el momento en que se producirán las piezas. El autor también establece que encuentran tres factores que afectan significativamente el rendimiento del modelo de investigación. Son: la complejidad de las partes componentes, el número de niveles de la estructura del producto y la capacidad del sistema (o, en otras palabras, las cantidades de las piezas componentes que se fabricarán).

\section{A. Incertidumbre en el Costo Unitario de Un Producto hasta la Distribución al Cliente}

El objetivo de este trabajo es presentar una metodología para determinar el costo unitario de un producto incluyendo la distribución al cliente en condiciones de incertidumbre. Se utiliza la herramienta de simulación para determinar los valores de las variables que intervienen desde que el proceso de planificación de los requerimientos de manufactura comienza hasta que el producto se distribuye al cliente final. Como resultado de esta interacción de variables se obtiene un conjunto de valores de costo, los cuales se comportan siguiendo un patrón de probabilidad determinado. Este patrón de probabilidad les servirá a las empresas para apoyar el proceso de toma de decisiones conociendo el valor más probable del costo unitario del producto hasta un valor pesimista del costo, con sus respectivas probabilidades asociadas. Este patrón de probabilidad encontrado servirá también a las empresas para conocer el impacto de la incertidumbre sobre el costo unitario del producto.

Cuando se calcula el costo unitario de un producto sobre la base de un requerimiento de producción que tiene su origen en un pronóstico de la demanda, el costo unitario obtenido corresponde al valor de los recursos de producción consumidos por esa producción en particular. $\mathrm{Si}$ los requerimientos de producción cambian de un periodo a otro, sería de esperar que los recursos de producción consumidos por el producto cambien también de un periodo a otro.

Esta variación de costos de un periodo a otro genera una incertidumbre para las empresas en estos tiempos, pues el control del costo unitario del producto es uno de los medios que tienen las empresas para generar una ventaja competitiva desde el punto de vista de las ganancias del negocio. De ahí la importancia para las empresas de conocer un posible rango en el que se movería el costo unitario del producto, y las respectivas probabilidades asociadas a estos valores de costo. Adicionalmente, cuando el producto se distribuye se genera un costo de distribución del producto, el cual depende de varios factores, entre ellos el tipo de flota de camión que distribuye el producto, las distancias recorridas y otros factores. Consecuentemente, determinar los rangos de variación del costo unitario hasta la distribución del producto sea de interés.

Katok (1996) [13] indica que las empresas manufactureras modernas deben operar de manera efectiva en un entorno dinámico y que un aspecto clave a considerar es la flexibilidad de fabricación o la capacidad de un sistema de fabricación para adaptarse a la incertidumbre en el entorno de producción.

Fildes, R; Kingsman, B (2011) [11] indican que el efecto de la incertidumbre de la demanda y el error de previsión en los costos unitarios y los niveles de servicio al cliente en la cadena de suministro, incluidos los sistemas de fabricación de tipo de Planificación de los Requerimientos de Materiales (MRP) es de importancia práctica porque los fabricantes gastan grandes sumas de dinero en sistemas de soporte de previsión de personal y compras para lograr pronósticos más precisos.

Consecuentemente, si se logran obtener pronósticos de demanda más precisos, se pueden obtener requerimientos de producción más precisos, que reducirían el impacto de la incertidumbre sobre el costo unitario del producto. 


\section{B. Metodología Para el Cálculo del Costo Unitario de Un Producto Hasta La Distribución al Cliente}

Delgado y Otros (2000) [6] indican que en la década de los 90, termina por imponerse la denominación ERP (Enterprise Resources Planning) y que, en gran parte, estos sistemas integrados de gestión empresarial pueden ser considerados como la extensión de los sistemas MRP, a partir de su uso en compras, producción, ingeniería y almacenes, a otras áreas de la empresa, como contabilidad y finanzas.

Bajo esta premisa, la figura 1 muestra una metodología de planificación de los recursos de manufactura para el cálculo del costo unitario del producto hasta su distribución al cliente.

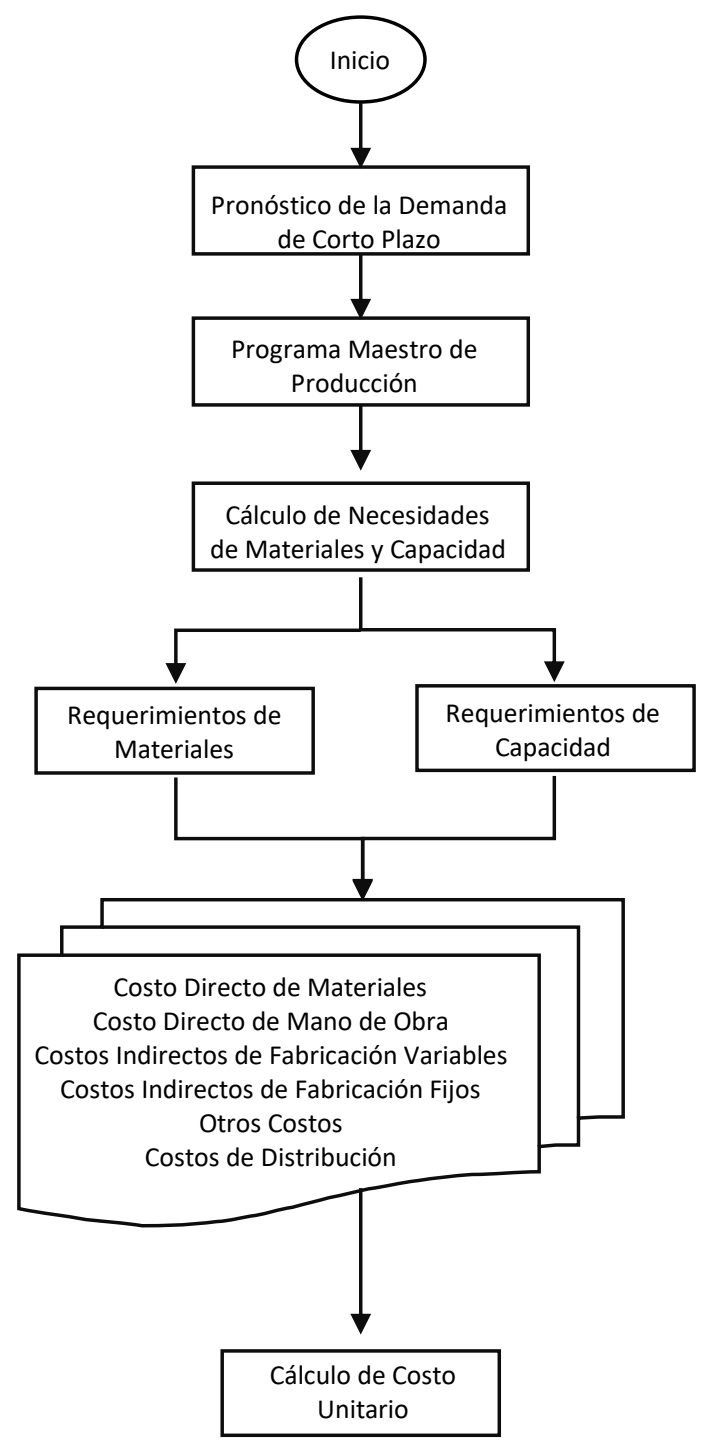

Fig. 1 Metodología del Plan de Recursos de Manufactura Para el Cálculo del Costo Unitario de un Producto hasta su Distribución.

\section{DEFINICION DEL CASO DE ESTUDIO}

Para la determinación del costo unitario de un producto en condiciones de incertidumbre se seleccionó un producto que consta de un ensamble de dos componentes principales, uno comprado y otro producido. El horizonte de planeación del programa maestro de producción se estableció en 12 periodos semanales. El producto final se denota por Parte P000000100, y el componente producido por Parte P000000120. El componente producido es a su vez un subensamble de otros dos componentes comprados. La lista de materiales y la secuencia de operaciones de las partes producidas, respectivamente, se muestran en las tablas 1 y 2 . Los componentes 000000110, 000000121 y 000000122 se compran.

TABLA 1 LISTA DE MATERIALES DEL PRODUCTO 1

\begin{tabular}{|c|c|c|}
\hline Parte & SubParte & Cantidad \\
\hline $\mathrm{P} 000000100$ & $\mathrm{P} 000000110$ & 2 \\
\hline $\mathrm{P} 000000100$ & $\mathrm{P} 000000120$ & 1 \\
\hline $\mathrm{P} 000000120$ & $\mathrm{P} 000000121$ & 1 \\
\hline $\mathrm{P} 000000120$ & $\mathrm{P} 000000122$ & 2 \\
\hline
\end{tabular}

TABLA 2

SECUENCIA DE OPERACIONES PARA LAS PARTES PRODUCIDAS

\begin{tabular}{|c|c|c|}
\hline \multirow{2}{*}{ Operación } & Parte P000000100 & Parte P000000120 \\
\cline { 2 - 3 } & Estación de Trabajo & Estación de Trabajo \\
\hline 01 y 04 & 03 & 01 \\
\hline 02 y 05 & 02 & 02 \\
\hline 03 y 06 & 03 & 01 \\
\hline
\end{tabular}

\section{Metodología de Simulación Para el Cálculo DEL COSTO UNITARIO}

La figura 2 resume la metodología de simulación para la determinación del costo unitario de un producto hasta su distribución al cliente.

El desarrollo de la simulación se realizó sobre la base de la metodología simplificada del cálculo de recursos de capacidad presentada por Domínguez Machuca y otros (1995), [7]. El plan de recursos de capacidad está contenido dentro de la metodología del plan de recursos de manufactura mostrado en la figura1. La simulación se realizó en Microsoft Office Excel 2016, plataforma sobre la cual se desarrollaron las macros correspondientes para llevar a cabo el proceso de simulación.

La metodología presentada en la figura 2 establece que para realizar la simulación se debe proceder a la generación de las variables aleatorias de cada una de las partes que intervienen en el sistema.

$1^{\text {th }}$ LACCEI International Multi-Conference for Engineering, Education, and Technology: "Innovation in Education and Inclusion”, 19-21 July 2018, Lima, Peru. 


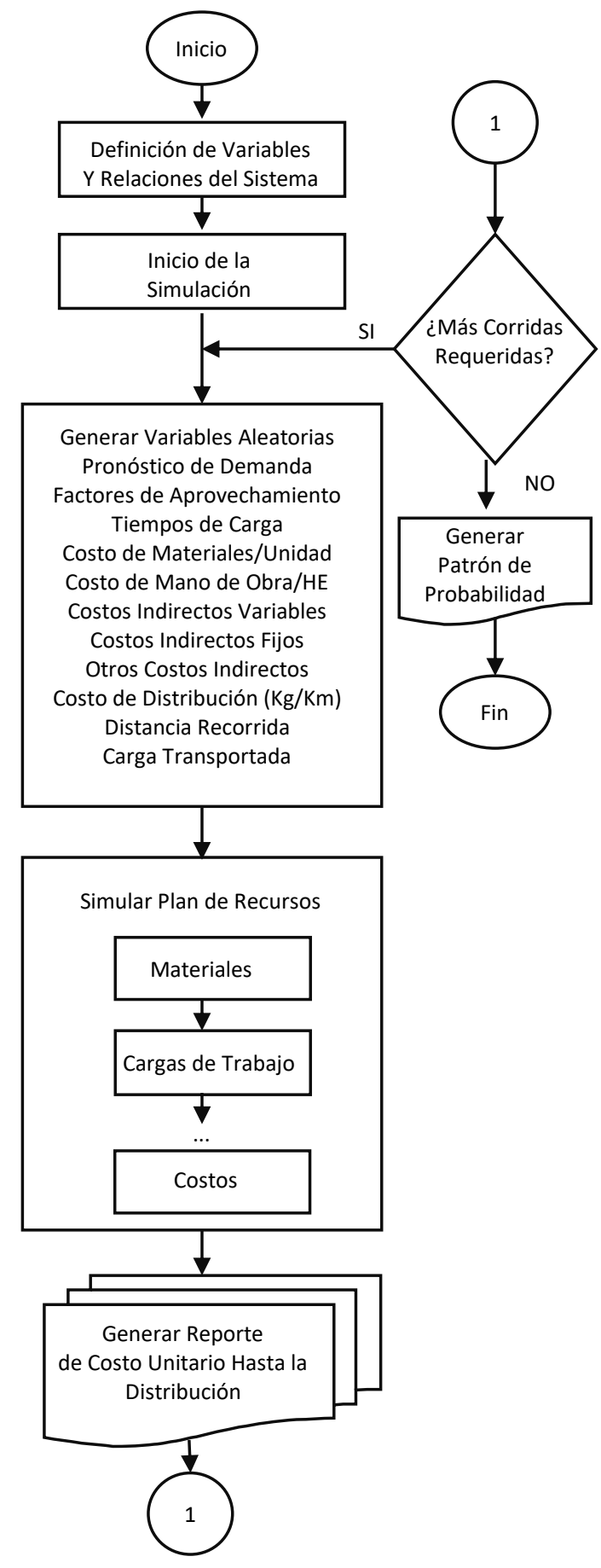

Fig. 2 Metodología de Simulación del Plan de Recursos de Manufactura Hasta su Distribución Al Cliente.

Para la especificación de los patrones de probabilidad de las diferentes variables que intervienen en el proceso de planificación se puede utilizar uno de los siguientes dos enfoques: 1) realizar las correspondientes pruebas de bondad de ajuste a partir de los datos históricos de las variables si es que la empresa dispone de tales datos para estas variables; 2) utilizar la distribución de probabilidad triangular, tomando como base un valor mínimo, un valor más probable y un valor máximo de las ventas de la empresa ó utilizar una distribución uniforme que requiere únicamente un valor máximo y un valor mínimo de las ventas del periodo, si es que únicamente se dispone de información por experiencia provenientes del departamento comercial de la empresa.

El sitio web de la compañía Palisade [18] indica que las variables que se pueden describir con una distribución triangular son el historial de ventas pasadas por unidad de tiempo y los niveles de inventario. Adicionalmente, el sitio también establece que las variables que se distribuyen de forma uniforme son los costos de manufacturación o los ingresos por las ventas futuras de un nuevo producto. Bajo este segundo enfoque se utilizaron las distribuciones de probabilidad triangular y uniforme.

En consecuencia, la tabla 3 muestra los patrones de probabilidad a simular para el plan de pronósticos de la demanda de corto plazo. Se presentan los patrones para cada uno de los próximos doce periodos del plan maestro de producción.

TABLA 3

DISTRIBUCIONES DE PROBABILIDAD TRIANGULARES PARA LOS PRONÓSTICOS DE LA DEMANDA DE CORTO PLAZO

\begin{tabular}{|c|c|c|c|}
\hline \multirow{2}{*}{ Semana } & \multicolumn{3}{|c|}{ Distribución Triangular } \\
& Pronósticos de la Demanda \\
\cline { 2 - 4 } & Mínimo & Más Probable & Máximo \\
\hline 1 & 40 & 60 & 80 \\
\hline 2 & 45 & 70 & 95 \\
\hline 3 & 50 & 75 & 100 \\
\hline 4 & 60 & 80 & 110 \\
\hline 5 & 70 & 90 & 120 \\
\hline 6 & 85 & 95 & 105 \\
\hline 7 & 50 & 80 & 120 \\
\hline 8 & 85 & 105 & 125 \\
\hline 9 & 80 & 95 & 115 \\
\hline 10 & 90 & 120 & 150 \\
\hline 11 & 100 & 120 & 140 \\
\hline 12 & 100 & 130 & 150 \\
\hline
\end{tabular}

La tabla 4 muestra los patrones de probabilidad a simular para el porcentaje de aprovechamiento de los materiales en cada una de las estaciones de trabajo del proceso de producción. Se sigue la misma premisa presentada para los datos de la tabla3.

Las tablas 5 y 6 muestran el patrón de probabilidad de los tiempos de carga de las operaciones para los artículos producidos. Los patrones de probabilidad normales se pueden obtener de las pruebas de bondad de ajuste realizadas sobre los tiempos de procesamiento de las operaciones en cada una de las estaciones de trabajo correspondientes.

$1^{\text {th }}$ LACCEI International Multi-Conference for Engineering, Education, and Technology: "Innovation in Education and Inclusion”, 19-21 July 2018, Lima, Peru. 
TABLA 4

DISTRIBUCIONES DE PROBABILIDAD PARA EL PORCENTAJE DE APROVECHAMIENTO DE MATERIALES

\begin{tabular}{|c|c|c|c|}
\hline \multirow{2}{*}{ Operación } & \multirow{2}{*}{ Estación de Trabajo } & \multicolumn{2}{|c|}{$\begin{array}{c}\text { Distribución Uniforme } \\
\% \text { de Aprovechamiento }\end{array}$} \\
\cline { 3 - 4 } & & Mínimo & Máximo \\
\hline 01 & 03 & 0.93 & 0.97 \\
\hline 02 & 02 & 0.97 & 0.99 \\
\hline 03 & 03 & 0.94 & 0.98 \\
\hline 04 & 01 & 0.89 & 0.92 \\
\hline 05 & 02 & 0.95 & 0.98 \\
\hline 06 & 01 & 0.94 & 0.96 \\
\hline
\end{tabular}

TABLA 5

DISTRIBUCIÓN DE PROBABILIDAD DEL TIEMPO DE CARGA DE LAS OPERACIONES PARTE P000000100

\begin{tabular}{|c|c|c|}
\hline \multirow{2}{*}{ Operación } & \multicolumn{2}{|c|}{$\begin{array}{c}\text { Parte P000000100 } \\
\text { Distribución Normal }\end{array}$} \\
\cline { 2 - 3 } & Media & $\begin{array}{c}\text { Desviación } \\
\text { Estándar }\end{array}$ \\
\hline 01 & 0.088 & 0.005 \\
\hline 02 & 0.023 & 0.003 \\
\hline 03 & 0.034 & 0.003 \\
\hline
\end{tabular}

TABLA 6

DISTRIBUCIÓN DE PROBABILIDAD DEL TIEMPO DE CARGA DE LAS OPERACIONES PARTE P000000120

\begin{tabular}{|c|c|c|}
\hline \multirow{2}{*}{ Operación } & \multicolumn{2}{|c|}{$\begin{array}{c}\text { Parte P000000120 } \\
\text { Distribución Normal }\end{array}$} \\
\cline { 2 - 3 } & Media & $\begin{array}{c}\text { Desviación } \\
\text { Estándar }\end{array}$ \\
\hline 04 & 0.087 & 0.005 \\
\hline 05 & 0.085 & 0.007 \\
\hline 06 & 0.076 & 0.004 \\
\hline
\end{tabular}

La tabla 7 muestra los patrones de probabilidad del costo de la mano de obra por hora estándar (He) de trabajo.

TABLA 7

DISTRIBUCIONES DE PROBABILIDAD DEL COSTO DE LA MANO DE OBRA POR HORA ESTÁNDAR (HE) DE TRABAJO

\begin{tabular}{|c|c|c|c|}
\hline \multirow{2}{*}{ Estación de Trabajo } & \multicolumn{3}{|c|}{ Distribución Triangular } \\
& Costo de la Mano de Obra/He \\
\cline { 2 - 4 } & Mínimo & Más Probable & Máximo \\
\hline 01 & 1650 & 1800 & 1950 \\
\hline 02 & 1200 & 1400 & 1600 \\
\hline 03 & 1200 & 1500 & 1800 \\
\hline
\end{tabular}

Domínguez Machuca y otros (1995), [7] indican que los sistemas de planificación de los recursos de manufactura diferencian como mínimo entre costos de materiales y mano de obra como costos directos. Los demás costos, indican los autores, los subdividen en costos indirectos de fabricación variables, costos indirectos de fabricación fijos y otros costos.

La tabla 8 presenta las distribuciones de probabilidad relacionadas con los costos fijos asociados al producto y al proceso de producción.

TABLA 8

DISTRIBUCIONES DE PROBABILIDAD DE LOS COSTOS FIJOS EN EL HORIZONTE DE PLANEACIÓN (HP)

\begin{tabular}{|c|c|}
\hline Concepto & Estación de Trabajo 01 \\
\hline Fabricación Variable/ Hora Estándar & Uniforme $(4000,5000)$ \\
\hline Fabricación Fijo en HP & Uniforme $(180000,220000)$ \\
\hline Otros Costos en HP & Triangular $(55000,60000,65000)$ \\
\hline Concepto Por Costo Fijo & Estación de Trabajo 02 \\
\hline Fabricación Variable/ Hora Estándar & Uniforme (3000,4000) \\
\hline Fabricación Fijo en HP & Uniforme $(120000,180000)$ \\
\hline Otros Costos en HP & Triangular (35000,40000,45000) \\
\hline & \\
\hline Concepto Por Costo Fijo & Estación de Trabajo 03 \\
\hline Fabricación Variable/ Hora Estándar & Uniforme (2000,3000) \\
\hline Fabricación Fijo en HP & Uniforme (175000,220000) \\
\hline Otros Costos en HP & Triangular (27000,30000,32500) \\
\hline
\end{tabular}

La tabla 9 muestra los datos de dimensionamiento del lote provenientes del registro de inventarios.

TABLA 9

DATOS DEL SEGMENTO MAESTRO DE INVENTARIOS

\begin{tabular}{|c|c|c|c|}
\hline Parte & $\begin{array}{c}\text { Método de } \\
\text { Dimensionado } \\
\text { del Lote }\end{array}$ & $\begin{array}{c}\text { Inventario de } \\
\text { Seguridad }\end{array}$ & $\begin{array}{c}\text { Tiempo de } \\
\text { Suministro }\end{array}$ \\
\hline P000000100 & EOQ =180 & 50 & 2 semanas \\
\hline P000000120 & Lote a Lote & 0 & 3 semanas \\
\hline
\end{tabular}

La tabla 10 muestra los costos asociados con los artículos comprados.

TABLA 10

DISTRIBUCIONES DE PROBABILIDAD DE LOS COSTOS DE LOS MATERIALES COMPRADOS

\begin{tabular}{|l|c|c|c|}
\hline \multirow{2}{*}{ Articulo } & \multicolumn{3}{|c|}{$\begin{array}{c}\text { Distribución Triangular } \\
\text { Costo de la Mano de Obra/He }\end{array}$} \\
\cline { 2 - 4 } & Mínimo & Más Probable & Máximo \\
\hline P000000110 & 240 & 350 & 660 \\
\hline P000000121 & 245 & 250 & 255 \\
\hline P000000122 & 380 & 420 & 460 \\
\hline
\end{tabular}

El costo de distribución en unidades monetarias por kilogramo por kilómetro recorrido se muestra en la tabla 11.

Para establecer este costo se tomó de referencia un estudio realizado en Argentina titulado "Costos y Precios del Transporte de Cargas "[5].

16 $^{\text {th }}$ LACCEI International Multi-Conference for Engineering, Education, and Technology: "Innovation in Education and Inclusion", 19-21 July 2018, Lima, Peru. 
TABLA 11

DISTRIBUCIÓN DE PROBABILIDAD DEL COSTO DE DISTRIBUCIÓN DEL PRODUCTO

\begin{tabular}{|c|c|c|c|}
\hline \multirow{2}{*}{} & \multicolumn{3}{|c|}{ Distribución Triangular } \\
& Costo Por Kilogramo Por Kilómetro Recorrido \\
\cline { 2 - 4 } & Mínimo & Más Probable & Máximo \\
\hline Costo de Distribución & 0.040 & 0.046 & 0.050 \\
\hline
\end{tabular}

\section{MODELO DE SIMULACION}

\section{A. Construcción del Modelo En Hoja Electrónica Excel}

Para la obtención de los resultados del costo estándar del producto hasta su distribución al cliente, se simuló el proceso de tres pasos mostrado en la figura 2. En primera instancia se simuló el plan de materiales (MRP) a partir de la simulación de las necesidades brutas de demanda y la posterior explosión de materiales. La tabla 12 muestra un extracto de la hoja electrónica correspondiente al MRP, con sus respectivas fórmulas.

TABLA 12

HOJA ELECTRÓNICA EXCEL PARA LA PLANIFICACIÓN DEL REQUERIMIENTO DE MATERIALES MRP

\begin{tabular}{|c|c|c|c|c|c|c|c|c|}
\hline 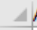 & $\mathrm{AO}$ & AP & $A Q$ & AR & AS & AT & \multicolumn{2}{|c|}{$A U$} \\
\hline \multicolumn{9}{|l|}{18} \\
\hline 20 & \multirow[b]{2}{*}{ Articulo } & \multirow[b]{2}{*}{ Nivel } & \multirow[b]{2}{*}{ SS } & \multirow[b]{2}{*}{ Conceptos } & \multicolumn{4}{|c|}{ Periodo } \\
\hline 21 & & & & & 1 & 2 & ... & 12 \\
\hline 22 & \multirow{6}{*}{ P000000100 } & \multirow{6}{*}{0} & \multirow{6}{*}{$=+$ AD 60} & $\mathrm{NB}$ & $=\operatorname{SI}((\mathrm{AS} 6-\mathrm{AS} 5) /(\mathrm{AS}$ & $=\operatorname{SI}(($ AT $6-A T 5)$ & . & $=\mathrm{SI}(\mathrm{BE} 6-\mathrm{BE} 5) /(\mathrm{BE}$ \\
\hline 23 & & & & Disp & $=A D 69$ & $=A S 23+A S 24-A S$ & $\ldots$ & $=\mathrm{BD} 23+\mathrm{BD} 26-\mathrm{BD} 2$ \\
\hline 24 & & & & RP & $=A E 69$ & & $\ldots$ & \\
\hline 25 & & & & $\mathrm{NN}$ & $=S I$ AS22-(AS23-S. & $=\operatorname{SI}(\mathrm{AT} 22-(\mathrm{AT} 23$ & $\ldots$ & $=\mathrm{SI}(\mathrm{BE} 22-(\mathrm{BE} 23-\mathrm{S}$. \\
\hline 26 & & & & RPP & $=S I$ AS $22-($ AS $23-S$. & $=$ SI (AT22-(AT2) & $\ldots$ & $=\mathrm{SI}(\mathrm{BE} 22-(\mathrm{BE} 23-\mathrm{S}$ \\
\hline 27 & & & & PP & =REDONDEAR.M & $=$ REDONDEAR. & $\ldots$ & =REDONDEAR.MA \\
\hline 34 & \multirow{6}{*}{ P000000120 } & \multirow{6}{*}{1} & \multirow{6}{*}{$=+\mathrm{AD} 62$} & $\mathrm{NB}$ & $=A . S 27+$ A.S33 & $=$ AT $27+$ AT 33 & . & $=\mathrm{BE} 27+\mathrm{BE} 33$ \\
\hline 35 & & & & Disp & $=A D 71$ & $=\operatorname{SI}($ AS35+AS36 & $\ldots$ & $=\mathrm{SI}(\mathrm{BD} 35+\mathrm{BD} 36-\mathrm{B}$ \\
\hline 36 & & & & $R P$ & & 113 & $\ldots$ & \\
\hline 37 & & & & $\mathrm{NN}$ & $=$ SI(AS34-(AS35-S. & $=$ SI (AT34-(AT3) & $\ldots$ & $=$ SI (BE34-(BE35-S. \\
\hline 38 & & & & RPP & $=\operatorname{SI}(\mathrm{A} S 37>0 ; \mathrm{AS} 37 ;$ & $=\operatorname{SI}(($ AT34- - AT3 & $\ldots$ & $=\operatorname{SI}(\mathrm{BE} 37<=0 ; 0 ; \mathrm{BE}$ \\
\hline 39 & & & & PP & $=$ =REDONDEAR.M & $=$ REDONDEAR.I & $\ldots$ & $=$ REDONDEAR.MA \\
\hline
\end{tabular}

Notación:

NB: Necesidades Brutas

Disp: Disponibilidades

RP: Recepciones Programadas

En segunda instancia se simuló el plan de requerimientos de capacidad para cada una de las estaciones de trabajo (ET) que intervienen en el proceso de producción. Se utilizó un procedimiento simplificado propuesto por Domínguez Machuca [7] que garantiza que el plan simulado es factible. El plan de capacidad se desarrolló para un horizonte de planeación de 12 periodos semanales.

La tabla 13 muestra una sección de la hoja electrónica Excel correspondiente.
TABLA13

HOJA ELECTRÓNICA EXCEL PARA LA PLANIFICACIÓN DE LOS REQUERIMIENTOS DE CAPACIDAD (CRP)

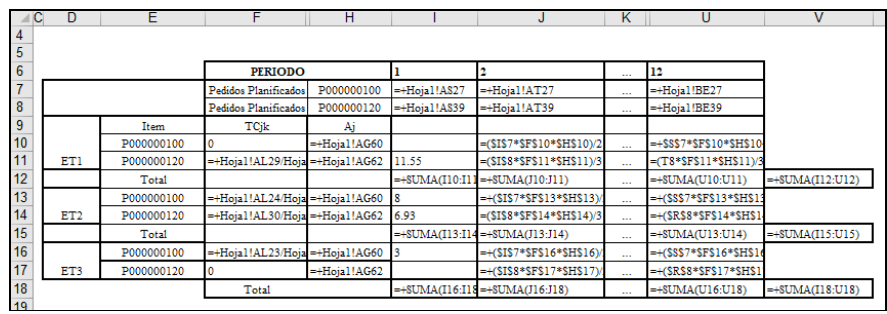

En tercera instancia se simuló el costo unitario. La tabla 14 muestra una parte de la hoja correspondiente para la simulación del cálculo de los costos unitarios para cada lista de materiales. Se incluyen los costos simulados de los materiales comprados, así como los costos de la mano de obra y los costos indirectos de fabricación variables, y fijos, así como otros de fabricación. Al final de la tabla se muestra el costo de distribución del producto, bajo el supuesto que el camión recorre 100 kilómetros y que el producto tiene un peso empacado de 15 kilogramos.

TABLA14

HOJA ELECTRÓNICA EXCEL PARA EL CÁLCULO DEL COSTO UNITARIO ESTÁNDAR DEL PRODUCTO P000000100

\begin{tabular}{|c|c|c|c|c|c|c|c|c|c|}
\hline & B C & D & E & $\mathrm{F}$ & G & H & I & $J$ & K \\
\hline $\begin{array}{l}6 \\
7\end{array}$ & Nivel & & Item & $\begin{array}{l}\text { Costo } \\
\text { Material }\end{array}$ & Costo & Costo & ecto & Otros Costos & Costo Total \\
\hline 8 & & P000000110 & Costo comprado & $=+\operatorname{SI}((\mathrm{N} 8-\mathrm{M} 8)$ & & & & & \\
\hline 9 & & & Costo unitar & $=+V C U$ & 0 & 0 & 0 & 10 & $=+$ SUMA $(F$ \\
\hline & 2 & P000000121 & Costo comprado & +SI((N10-M1 & & & & & \\
\hline 11 & & & Costo unitaric & $=+F 10$ & 0 & 0 & 0 & 0 & $=+$ SUMA $(F$ \\
\hline $\begin{array}{l}12 \\
13\end{array}$ & & P000000122 & $\begin{array}{l}\text { Costo comprado } \\
\text { Costo unitario }\end{array}$ & $\begin{array}{l}=+\mathrm{SI}((\mathrm{N} 12-\mathrm{M} 1 \\
=+\mathrm{F} 12\end{array}$ & 0 & 10 & 10 & 0 & \\
\hline 14 & & & Costo $211 / \mathrm{A}_{112}$ & $=+F 11 /$ Hoja 11 & & - & & & \\
\hline 15 & & & Costo $212 \times 2 / A_{112}$ & $=+\mathrm{F} 13^{*} 2 / \mathrm{Hoja}$ & & - & & & \\
\hline 16 & 1 & P0000000120 & Costo 07 & - & $=+$ Hoja2.:J7 & $=+\mathrm{Hoja} 2 \mathrm{2N} 72 / \mathrm{Hoj}$ & $=+\mathrm{Hojaj}_{2} 2.773 / \mathrm{Ho}$ & D =+Hoja2.1.J4 4/HC & \\
\hline & & & Costo 08 & : & =+Hoja2:K7 & $=+\mathrm{Hoja} 2 / \mathrm{K} 72 / \mathrm{Ho}$ & $=+H o j a 2 ! K 73 / H o$ & ja2!K74/H & \\
\hline 19 & & & Costo Unitario & $=+$ SUMA(F 14 & $=+$ SUMA $(G$ & $=+S U M A(H 14: H$ & $\begin{array}{l}=++5 U M A(14: 118 \\
=+S\end{array}$ & $\begin{array}{l}=+\operatorname{SUMA}(\mathrm{J14}: \mathrm{J} \\
\mathrm{l}\end{array}$ & $=+S U M A(F$ \\
\hline 20 & & & Costo 112/Ap1 & $=+F 19 /$ Hoja 1 & $y=+G 19 /$ Hoja & $=+\mathrm{H} 19 / \mathrm{Hoj}$ a $1 / \mathrm{A} \mathrm{J}$ & $=+119 /$ Hoja 1 A A 2 & $2=+\mathrm{J} 19 / \mathrm{Hoj}_{1} 1 \mathrm{~A}_{\mathrm{A}}$ & \\
\hline 21 & & & Costo $111^{*} 2 / A_{p_{1}}$ & $=+F 9 * 2 / \mathrm{Hoja} 1$ & & & & & \\
\hline 22 & 10 & P0000000100 & Costo 01 & 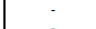 & $=+$ +Hoja21:F 7 & $=+\mathrm{Hoja}_{2} / \mathrm{F} 72 / \mathrm{Ho}$ & $=+H_{0 j a 21 / F 73 / H o}$ & $0=+H 0 j a 21 F 74 / H$ & \\
\hline & & & $\mid \begin{array}{l}\text { Costo } 02 \\
\text { Costo } 03\end{array}$ & : & $\begin{array}{l}=+H o j a 2 \cdot G 7 \\
=+H o i a 2 H 7\end{array}$ & 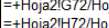 & 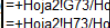 & & \\
\hline $\begin{array}{l}24 \\
25\end{array}$ & & & $\begin{array}{l}\text { Costo } 03 \\
\text { Costo Unitario }\end{array}$ & $=+\operatorname{SUMA}(\mathrm{F} 20$ & 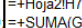 & 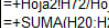 & 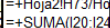 & $\begin{aligned} g=+H o j a 21 \\
4 \\
=+ \text { SUMA }\end{aligned}$ & $=+\operatorname{SUMA}(\mathrm{F}$ \\
\hline 26 & & & & & & & DIS & & \\
\hline $\begin{array}{l}27 \\
28\end{array}$ & & & & & & & $\begin{array}{l}\text { PESO } \\
\text { COSTC }\end{array}$ & & $=++\ldots$ \\
\hline 29 & & & & & & & $\begin{array}{l}\text { COSTO CARGA } \\
\text { COR }\end{array}$ & $A=(J 26$ & \\
\hline
\end{tabular}

\section{B. Número de Réplicas}

Con base en una muestra preliminar de cincuenta corridas del simulador, se determinó que para alcanzar un nivel de confianza de $95 \%$ y un error máximo de estimación de tres unidades monetarias en la estimación del costo unitario promedio del producto hasta su distribución al cliente final se deben realizar un total de diez mil corridas del simulador. El costo unitario en cada corrida se obtuvo con base en cinco réplicas del simulador.

16 $^{\text {th }}$ LACCEI International Multi-Conference for Engineering, Education, and Technology: "Innovation in Education and Inclusion", 19-21 July 2018, Lima, Peru. 


\section{Desarrollo de Macros}

Para llevar a cabo el número de corridas especificado en el apartado B) anterior se desarrolló el macro denominado "SimCostEst" en lenguaje de Visual Basic para Excel. El código generó los datos en un total de cinco columnas. En la columna 1 se generó el costo total por unidad de producto. En las restantes cuatro columnas se generaron los costos desagregados. Estos son el costo de los materiales comprados, el costo de mano de obra obtenido a partir de la carga de los centros de trabajo y los costos indirectos de fabricación divididos en costos indirectos de fabricación variables, costos indirectos de fabricación fijos y otros costos indirectos.

\section{RESULTADOS OBTENIDOS}

La figura 3 muestra el patrón de probabilidad del costo unitario promedio simulado del producto. Una prueba de bondad de ajuste indica que hay suficiente evidencia para creer que el costo unitario promedio sigue una distribución de probabilidad normal.

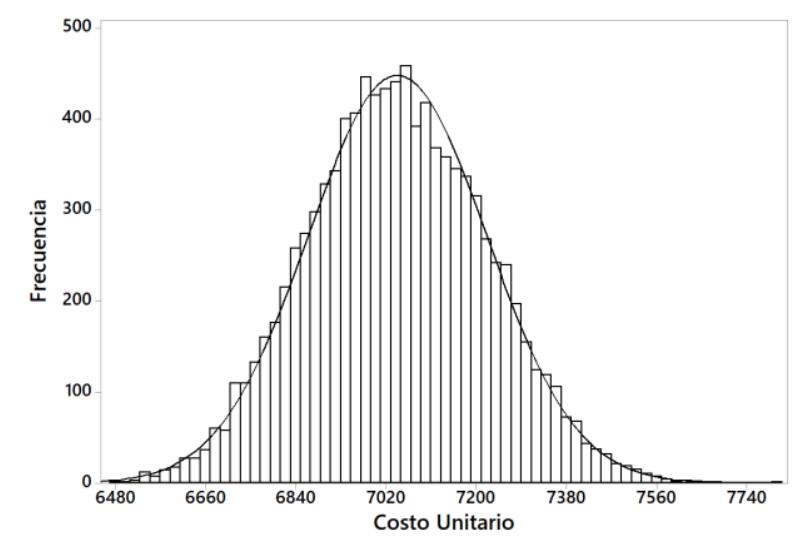

Fig.3 Patrón de Probabilidad del Costo Unitario del Producto Hasta La Distribución al Cliente.

El intervalo de confianza de $95 \%$ para el costo unitario promedio indica que este es un valor que está entre 7039.49 y 7046.48 unidades monetarias. El valor más probable del costo es de 7042.98 unidades monetarias con una desviación estándar de 178.18 unidades. Estos valores se muestran en la figura 4 .

La tabla 15 resume el costo unitario promedio, así como la desviación estándar y el intervalo de confianza del 95\% para los costos directos de materiales y mano de obra.

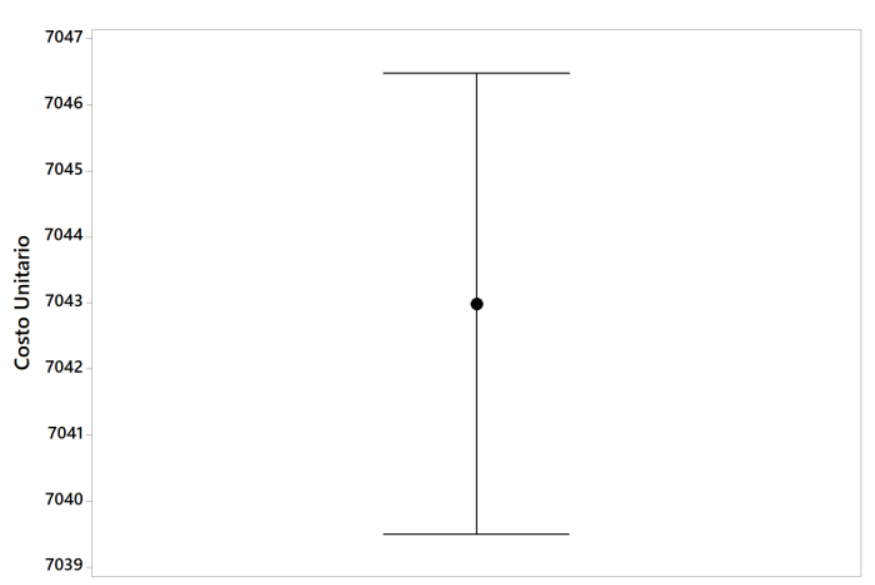

Fig.4 Intervalo de Confianza de 95\% Para el Costo Unitario.

TABLA15

COSTOS DIRECTOS DEL PRODUCTO P000000100

\begin{tabular}{|l|c|c|c|}
\hline Costos Directos & Promedio $\mu$ & $\begin{array}{c}\text { Desviación } \\
\text { Estándar }\end{array}$ & IC de $95 \%$ para $\mu$ \\
\hline Materiales & 2925.85 & 68.73 & $2924.51 ; 2927.20$ \\
\hline Mano de Obra & 752.84 & 32.04 & $752.21 ; 753.47$ \\
\hline
\end{tabular}

La tabla 16 resume el costo promedio, la desviación estándar y el intervalo de confianza del $95 \%$ para los costos indirectos de fabricación y distribución.

TABLA16

COSTOS INDIRECTOS DEL PRODUCTO P000000100

\begin{tabular}{|c|c|c|c|}
\hline $\begin{array}{c}\text { Costos Indirectos de } \\
\text { Fabricación }\end{array}$ & $\begin{array}{c}\text { Promedio } \\
\mu\end{array}$ & $\begin{array}{c}\text { Desviación } \\
\text { Estándar }\end{array}$ & IC de 95\% para $\mu$ \\
\hline Variables & 1714.24 & 100.32 & $1712.28 ; 1716.21$ \\
\hline Fijos & 536.46 & 25.69 & $535.96 ; 536.96$ \\
\hline Otros Costos & 1046.11 & 27.70 & $1045.57 ; 1046.66$ \\
\hline Costo de Distribución & 67.48 & 2.53 & $67.43 ; 67.53$ \\
\hline
\end{tabular}

La tabla 17 muestra el percentil 5 y 95 para el costo unitario del producto hasta su distribución al cliente. Estos costos corresponden al escenario optimista y pesimista del costo.

TABLA17

PERCENTILES 5 Y 95 DEL COSTO UNITARIO DEL PRODUCTO $\mathrm{P} 000000100$

\begin{tabular}{|l|l|l|}
\hline \multirow{2}{*}{ Costo Unitario } & $\begin{array}{l}\text { Percentil 5 } \\
\text { (u.m.) }\end{array}$ & $\begin{array}{l}\text { Percentil 95 } \\
\text { (u.m.) }\end{array}$ \\
\cline { 2 - 3 } & 6752.46 u.m. & 7339.72 u.m. \\
\hline
\end{tabular}

La tabla 18 presenta los percentiles 5 y 95 , en unidades monetarias, para los componentes del costo unitario.

16 ${ }^{\text {th }}$ LACCEI International Multi-Conference for Engineering, Education, and Technology: "Innovation in Education and Inclusion", 19-21 July 2018, Lima, Peru. 
TABLA18

PERCENTILES 5 Y 95 DE LOS COMPONENTES DEL COSTO UNITARIO DEL PRODUCTO P000000100.

\begin{tabular}{|l|c|c|}
\hline Costos & Percentil 5 & Percentil 95 \\
\hline Materiales & 2814.24 & 3040.75 \\
\hline Mano de Obra & 700.66 & 805.52 \\
\hline Fabricación Variables & 1551.30 & 1882.68 \\
\hline Fabricación Fijos & 496.15 & 577.05 \\
\hline Otros Costos Indirectos & 1024.57 & 1069.42 \\
\hline Distribución & 63.29 & 71.63 \\
\hline
\end{tabular}

\section{A. Comparación de Resultados Con Uso de Software Comercial de Simulación}

La figura 5 muestra los resultados obtenidos por el simulador Analytic Solver Plataform, el cual es una marca registrada de la compañía Frontline Systems. El simulador se corrió con el mismo número de réplicas con que se corrió el macro simulador SimCostEst. La tabla 19 muestra, como una forma de validación, una comparación entre los resultados obtenidos con el macro simulador SimCostEst y el simulador Analytic Solver Platform. Claramente se evidencia que no hay una diferencia significativa entre los resultados obtenidos por ambos procedimientos.

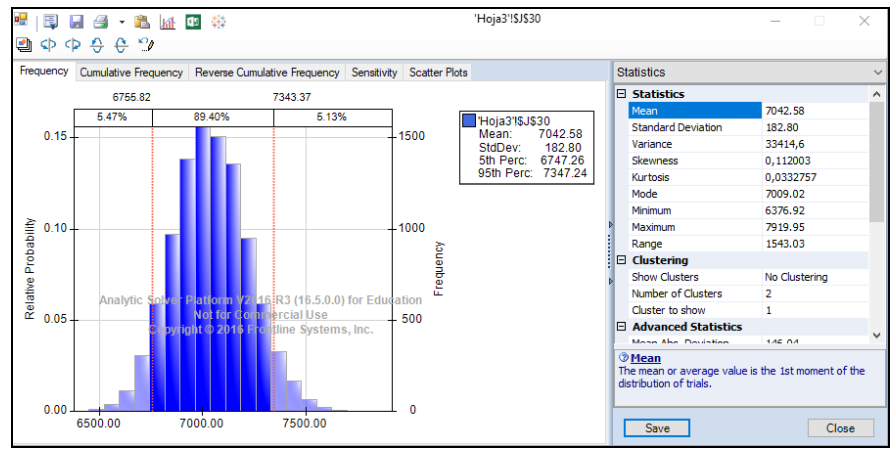

Fig.5 Resultados Obtenidos por el Simulador Analytic Solver Platform para el Cálculo del Costo Unitario.

TABLA19

COMPARACIÓN DE RESULTADOS CON EL SIMULADOR ANALYTIC SOLVER PLATFORM

\begin{tabular}{|l|c|c|}
\hline Concepto del Costo & $\begin{array}{c}\text { Simulador } \\
\text { SIMCOSTEST }\end{array}$ & $\begin{array}{c}\text { Analytic Solver } \\
\text { Platform }\end{array}$ \\
\hline Promedio & 7042.98 & 7042.58 \\
\hline Desviación Estándar & 178.18 & 182.80 \\
\hline Percentil 5 & 6752.46 & 6747.26 \\
\hline Percentil 95 & 7339.72 & 7347.24 \\
\hline
\end{tabular}

Finalmente, una ventaja substancial del uso del simulador Analytic Solver Platform es que no requiere programación, por lo que la obtención de resultados se realiza en un periodo de tiempo considerablemente más corto que si se requiere escribir código de macros para realizar la simulación. El programa dispone de funciones generadoras de números aleatorios para un buen número de distribuciones de probabilidad, así como la especificación de manera simple de las variables del sistema que se quieren simular. Una ventaja adicional del software es la facilidad de parametrización de variables de entrada para estudiar el comportamiento de las variables de respuesta del sistema estudiado.

\section{ConClusiones}

1. El sistema de planificación de los requerimientos de manufactura es una excelente técnica para la determinación del costo estándar de un producto.

2. Los sistemas de planificación de los recursos de manufactura unifican la información de las diferentes áreas funcionales del negocio para la determinación de los costos estándares de los productos.

3. La simulación es una herramienta poderosa para determinar la variabilidad del costo estándar de producción cuando se presentan cambios en las variables de entrada que afectan este costo.

4. La simulación permite estudiar con gran detalle el comportamiento de los diferentes componentes del costo estándar de un producto.

5. La simulación permite determinar cuáles son los componentes del costo estándar que más variación están teniendo en el sistema de planificación de la producción.

6. Los costos estándar difieren ligeramente de los costos reales, por lo que se deben calcular de manera periódica las variaciones causadas por cambios en la cantidad de mano de obra y cambios en el costo de los materiales.

\section{REFERENCIAS}

[1] Accounting Tools: Standard Cost Overview https://www.accountingtools.com/articles/2017/5/14/standard-costing

[2] Baunton, M J. Computer Aided Production Management Conference; Wembley; London (UK); 30 Apr. 1985, (Apr 30, 1985).

[3] Breaking Down Manufacturing Resource Planning - MRP II https://www.investopedia.com/terms/m/manufacturing-resourceplanning.asp

[4] Chen, Jhitang Steve. Integration of Process Planning with MRP and Capacity Planning for Better Shop Production Planning and Control. Purdue University, ProQuest Dissertations Publishing, 1981. 8200758.

[5] Costos y Precios del Transporte de Cargas http://www.edutecne.utn.edu.ar/transporte/costos.pdf

[6] Delgado, J; Marín, F. Evolución en los sistemas de gestión empresarial. Del MRP al ERP. Economía Empresarial No. 331. 2000/I

http://www.minetad.gob.es/Publicaciones/Publicacionesperiodicas/Econ omiaIndustrial/RevistaEconomiaIndustrial/331/09.JOAQUIN\%20DEL GADO.pdf 
[7] Domínguez, M. García, S, Ruiz, A. Dirección de Operaciones. Aspectos Tácticos y Operativos de la Producción. Editorial Mc Graw Hill. 1995.

[8] El Análisis de las Variaciones de los Costos de Producción. http://www.monografias.com/trabajos30/costos-estandar/costosestándar.shtml\#defin

[9] How to Calculate the Total Manufacturing Price per Unit. http://smallbusiness.chron.com/calculate-total-manufacturing-price-per unit-65756.html

[10] Hung, Yi-Feng. Huang, Chuan-Che. Yeh, Ying. Real Time capacity requirement planning for make-to-order manufacturing with variable time-window orders. Computers \& Industrial Engineering. Feb2013, Vol. 64 Issue 2, p641-652. 12p.

[11] Fildes, R; Kingsman, B. Incorporating Demand Uncertainty and Forecast Error in Supply Chain Planning models. The Journal of the Operational Research Society; Abingdon Vol. 62, Iss. 3, (Mar 2011): 483-500.

[12] Jinks, Stuart. Integrating Supply Chain Simulation, Component Geometry, and Unit Cost Estimation. University of Southampton (United Kingdom), ProQuest Dissertations Publishing, 2012. U600659.

[13] Katok, Elena. Planning Manufacturing Flexibility in an Uncertain Production Environment. The Pennsylvania State University, ProQuest Dissertations Publishing, 1996. 9716241

[14] MIME Enterprise: Improve the Company Performance. http://www.ipol.no/en/MRPII.asp

[15] MRP Versus MRP II. https://bizfluent.com/facts-7664435-difference-mrp-mrp-ii-systems.html

[16] Planificación de Recursos de Manufactura MRP II. http://whatis.techtarget.com/definition/Manufacturing-resource-planningMRP-II

[17] Peirleitner,A; Altendorfer,K; Felberbauer,T. Simulation Based Manufacturing System Improvement Focusing on Capacity and MRP Decisions - A Practical Case from Mechanical Engineering. The Institute of Electrical and Electronics Engineers, Inc. (IEEE) Conference Proceedings; Piscataway. 2017 inter Simulation Conference (WSC).

[18] Simulación Montecarlo http://www.palisade-lta.com/risk/simulacion_monte_carlo.asp

[19] Waterman, R; Rubin, D; Thomas, N; Gelman, A. Simulation Modeling for Cost Estimation. Seventh Conference on Postal and Delivery Economics: Current Directions in Postal Reform. June 23-26, 1999, Sintra, Portugal.

$16^{\text {th }}$ LACCEI International Multi-Conference for Engineering, Education, and Technology: "Innovation in Education and Inclusion", 19-21 July 2018, Lima, Peru. 